\title{
The Expression of mRNA for Calcitonin Gene-Related Peptide Receptors in a Mucosal Type Mast Cell Line, RBL-2H3
}

\author{
Yuki Kitabatake, Shunsuke Kawamura, Masamichi Yamashita, Kaori OKuYama, \\ Motoaki TAKAYANAGI, and Isao OHNO* \\ Department of Pathophysiology, Tohoku Pharmaceutical University; 4-4-1 Komatsushima, Aoba-ku, Sendai 981-8558, \\ Japan. Received December 8, 2003; accepted March 5, 2004; published online March 10, 2004
}

\begin{abstract}
Calcitonin gene-related peptide (CGRP) and adrenomedullin (ADM) belong to a calcitonin-family of regulatory peptides. Receptors for CGRP and ADM have been suggested to be present on both mucosal (MMC) and connective tissue (CTMC) type of mast cells, based on histamine release by these peptides. Recently, it was reported that mRNA for ADM receptors, but not for CGRP receptors, was expressed in rat peritoneal mast cells, a representative of type CTMC. However, mRNA expression for the receptors in MMC has not been studied yet. Therefore, we examined whether mRNAs encoding CGRP or ADM receptor subunit, RDC-1, calcitonin receptor-like receptor (CRLR), and receptor activity-modifying proteins (RAMPs) are present, and if so, whether their expression is modified by IgE receptor triggering, in a mucosal type mast cell line, rat basophilic leukemia (RBL-2H3) cells using RT-PCR. RBL-2H3 cells constitutively express mRNA for RDC-1, CRLR, RAMP3 but not that for RAMP1 and RAMP2, and IgE receptor triggering was shown neither to induce the gene expression of RAMP1 and RAMP2, nor to enhance that of RDC-1, CRLR or RAMP3. These results indicate that RBL-2H3 cells posses receptors for both CGRP and ADM, suggesting various functions of these peptides in physiological and pathophysiological conditions where mast cells of the mucosal type are involved.
\end{abstract}

Key words calcitonin gene-related peptide (CGRP); adrenomedullin; mast cell; RDC-1; calcitonin receptor-like receptor (CRLR); receptor activity-modifying protein (RAMP)

Calcitonin gene-related peptide (CGRP) and adrenomedullin (ADM) belong to a calcitonin-family of regulatory peptides. ${ }^{1)}$ CGRP is a 37-amino acid neuropeptide present throughout the central and peripheral nerve fibers coexisting with substance P. Neuropeptides were considered to be major mediators of neurogenic inflammation, since they display a wide range of peripheral effects such as vasodilation, plasma extravasation, and immunomodulation. ${ }^{1,2)}$ ADM is a 52-amino acid vasoactive polypeptide, which synthesized and secreted by non-neuronal cells such as endothelial cells and vascular smooth muscle cells ${ }^{3-5)}$ and also found in inflammatory cells including monocytes/macrophages, granulocytes, and lymphocytes. ${ }^{4,5)}$ To date, cloning studies have claimed several receptors for CGRP and ADM including RDC-1 and calcitonin receptor-like receptor (CRLR)/receptor activity- modifying protein (RAMP) heterodimers. ${ }^{6,7)}$ RDC-1, which was named by the authors, was originally cloned from dog thyroid $\mathrm{cDNA}^{8)}$ and has been cloned also in human and rodent. ${ }^{9}{ }^{9}$ RDC-1 binds CGRP with high affinity but is responsive to ADM. ${ }^{6}$ The CRLR can either function as a CGRP or ADM receptor depending on which member of three different RAMPs is co-expressed. Co-expression of RAMP1 and CRLR act as the CGRP receptors. By contrast, CRLR/RAMP2 or CRLR/RAMP3 receptor complex behaves as an ADM receptors. ${ }^{7,10)}$

Mast cells play an important role in the immunopathology of allergic reactions as well as in the pathogenesis of chronic inflammation. Activation of mast cells, dependent on or independent of immunoglobulin-E (IgE) receptors, leads to the release of a variety of inflammatory mediators such as histamine, 5-hydroxytriptamine, newly synthesized arachidonic acid metabolites, and cytokines. ${ }^{11,12)}$ Two classes of mast cells, the mucosal type (MMC) and connective tissue type (CTMC), have been described based on biological and functional properties. Rat basophilic leukemia (RBL-2H3) cells and rat peritoneal mast cells (PMCs) are examples of MMC and CTMC, respectively. ${ }^{13,14)}$ CGRP can induce histamine release from certain types of mast cells such as human skin mast cells, ${ }^{15}$ ) bronchoalveolar lavage (BAL) fluid mast cells, ${ }^{16)}$ and rat dural mast cells ${ }^{17)}$ but not from rat PMCs. ${ }^{17,18)}$ $\mathrm{ADM}$ has been reported to cause histamine release from rat PMCs. ${ }^{18)}$

Recently, rat PMCs were found to express mRNA for CRLR, RAMP2 and RAMP3, receptors for ADM, but not RAMP1 for CGRP. ${ }^{19)}$ However, the expression of these mRNAs in mucosal mast cells has not been determined yet. Therefore, we examined whether mRNAs encoding CGRP or ADM receptor subunit, RDC-1, CRLR and RAMPs are present and whether, if so, their expression is modified by $\operatorname{IgE}$ receptor triggering, in the rat basophilic leukemia cell line RBL-2H3 using RT-PCR.

\section{MATERIALS AND METHODS}

Culture of RBL-2H3 Cells RBL-2H3 cells (Japan Health Sci Found, Osaka, Japan) were cultured, passively sensitized and stimulated with dinitrophenol conjugated human serum albumin (DNP-HSA) as previously described. ${ }^{20)}$ Determinations of the degranulation of RBL-2H3 cells were performed as previously described. ${ }^{20)}$

RNA Extraction and RT-PCR Total RNA was isolated from non-sensitized RBL-2H3 cells, the sensitized cells before and after the stimulation as mentioned above, and homogenized lung tissues of Wistar rats (male, $300 \mathrm{~g}$ body weight, Japan SLC, Shizuoka, Japan) by RNeasy Minikit (QIAGEN Inc., Valencia, CA, U.S.A.) according to the manufacturer's protocols.

Total RNA was reverse transcribed and amplified with RTPCR beads (Amersham Biosci, Corp., Piscataway, NJ, U.S.A.) according to the manufacturer's protocols. Negative 
Table 1. Oligonucleotide Sequences

\begin{tabular}{ll}
\hline \hline Gene (base pair) & \multicolumn{1}{c}{ Primer sequence } \\
\hline RDC-1 $(354 \mathrm{bp})$ & \\
Forward: & 5'-GTGCAGCATAACCAGTGGCC-3' \\
Reverse: & 5'-AGCAAAACCCAAGATGACGGA-3' \\
CRLR $(403 \mathrm{bp})$ & \\
Forward: & 5'-AACAACAGCACGCATGAGAA-3' \\
Reverse: & 5'-ACCCCCAGCCAAGAAAATAA-3' \\
RAMP1 $(230 \mathrm{bp})$ & \\
Forward: & 5'-ACTGGGGAAAGACCATAGGGAG-3' \\
Reverse: & 5'-AGTCATGAGCAGTGTGACCGTA-3' \\
RAMP2 $(175 \mathrm{bp})$ & \\
Forward: & 5'-AGGTATTACAGCAACCTGCGGT-3' \\
Reverse: & 5'-ACATCCTCTGGGGGATCGGAGA-3' \\
RAMP3 $(416$ bp) & \\
Forward: & 5'-GTATGCGGTTGCAATGAGACA-3' \\
Reverse: & 5'-TCTTCTAGCTTGCCAGGCAC-3' \\
GAPDH ${ }^{a}(249 \mathrm{bp})$ & \\
Forward: & 5'-TGATGACATCAAGAAGGTGGTGAAG-3' \\
Reverse: & 5'-TCCTTGGAGGCCATGTAGGCCAT-3' \\
\hline
\end{tabular}

a) GAPDH: glyceraldehyde 3-phophate dehydrogenase.

controls were run in which RNA samples were replaced by RNAase-free water in the reactions. Each upstream and downstream specific primer (Fasmac Co., Ltd, Kanagawa, Japan) corresponding to the sequence of interest is shown in Table 1. The PCR reaction and the RT-PCR product were separated by electrophoresis as previously described. ${ }^{21,22)}$

Quantification of the Relative Abundance of mRNA The photographed PCR gel image was digitized by using a scanner. The density of the detected bands in the digitized image was determined by the public domain NIH imaging program. For all PCR samples, the density of an individual target mRNA band was divided by that of the corresponding GAPDH mRNA band to correct for differences in RNA loading.

Statistical Analysis Target/GAPDH ratios are presented as mean \pm S.D. Data were compared using analysis of variance (ANOVA) and Tukey test. The differences were considered significant at $p<0.05$.

\section{RESULTS AND DISCUSSION}

Agarose gel electrophoresis of the RT-PCR products from RBL-2H3 cells with the primers for RDC-1, CRLR, and RAMP3 showed single bands of 354, 403, and $416 \mathrm{bp}$, respectively (Fig. 1, lane 2), the sizes of which are identical to those observed in the RT-PCR products from rat lung tissues as a positive control (Fig. 1, lane 1). However, no PCR products with the primers for RAMP1, and RAMP2 were found from the RBL-2H3 cells (Fig. 1, lane 2).

Semi-quantification of the receptor mRNAs corrected by mRNA for GAPDH revealed no significant increase of RDC1, CRLR, or RAMP3 transcripts in RBL-2H3 cells either $4 \mathrm{~h}$ $(1.16 \pm 0.06,1.14 \pm 0.10$ and $1.22 \pm 0.23$, respectively) or $8 \mathrm{~h}$ $(1.32 \pm 0.14,1.37 \pm 0.20$ and $1.52 \pm 0.23$, respectively) after IgE receptor triggering, compared to those transcripts in the unstimulated cells $(1.38 \pm 0.11,1.42 \pm 0.11$ and $1.35 \pm 0.19$, respectively) (Fig. 2). Neither mRNA for RAMP1 nor RAMP2 was detected in RBL $2 \mathrm{H} 3$ cells at any time points after $\operatorname{IgE}$ receptor triggering (data not shown). IgE receptor triggering was confirmed by $\beta$-hexosaminidase release from

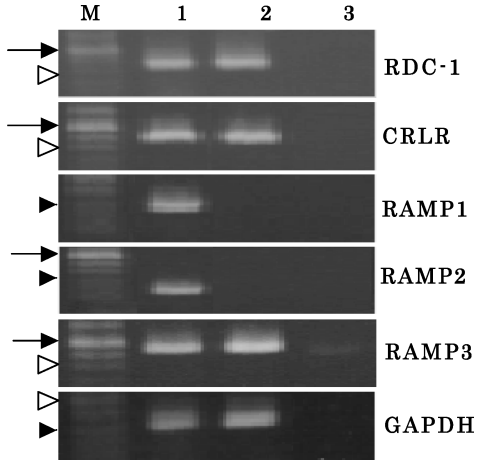

Fig. 1. Gel Electrophoresis of RT-PCR Products of RNA from RBL-2H3 Cells

RNA from rat lung tissues (lane 1) or RBL-2H3 cells (lane 2), and RNAase-free water (lane 3) were subjected to RT-PCR with primers for RDC-1, CRLR, RAMP3, and GAPDH. Photos are representatives of three independent RT-PCR experiments. M: a DNA molecular weight maker. The migration positions of 404 (arrows), 320 (arrow heads), and 240 (open arrow heads) bp fragments are indicated to the left.

(A)

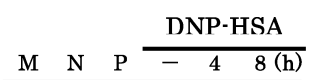

RDC-1

CRLR

RAMP3

GAPDH

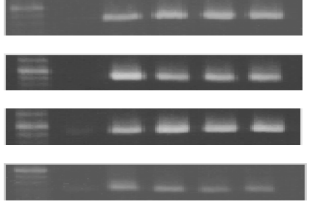

(B)

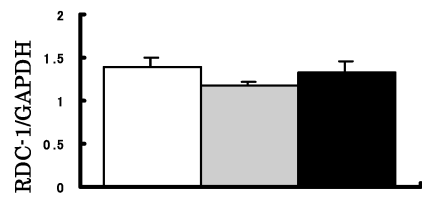

(C)

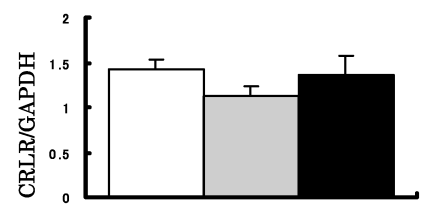

(D)

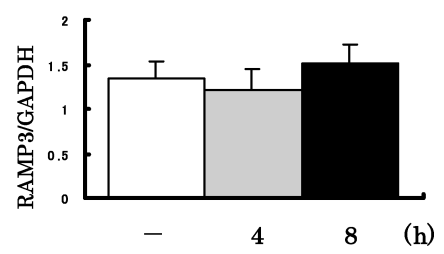

Fig. 2. Evaluation of RNA from RBL-2H3 Cells after IgE Receptor Triggering

RNA from rat lung tissues (P) or sensitized RBL-2H3 cells before (open columns) or 4 (hatched columns) and 8 (closed columns) h after the stimulation with the antigen, and RNAase-free water $(\mathrm{N})$ were subjected to RT-PCR with primers for RDC-1, CRLR, RAMP3, and GAPDH. Representative photos of gel electrophoresis of RT-PCR products and a molecular marker (M) are shown in (A). Relative levels of mRNA for RDC1 (B), CRLR (C), and RAMP3 (D) in RBL-2H3 cells before (-) or 4 and $8 \mathrm{~h}$ after the stimulation with the antigen were determined as described in Materials and Methods. The data are presented as mean \pm S.D. $(n=4)$. Bars show S.D.

sensitized RBL-2H3 cells after the addition of the specific antigen $(46.7 \pm 1.36 \%, n=4)$.

Recently, Matsushima et al. demonstrated that mRNAs of CRLR, RAMP2 and RAMP3, but not RAMP1, are expressed constitutively in rat $\mathrm{PMCs}^{19)}$ from which ADM causes histamine release. ${ }^{18)}$ CGRP can induce histamine release from human skin mast cells, ${ }^{15)}$ human BAL mast cells, ${ }^{16)}$ and rat dural mast cells. ${ }^{17)}$ However, the expression of mRNA for 
CGRP receptors in these mast cells has not been reported yet. The receptors for ADM and CGRP in the other phenotype, MMC, have not been examined yet. RDC-1 expression has not yet been reported in either phenotype. Our findings are similar to those of Matsushima et al. in the view of that the combination of CRLR with RAMP favors the receptors for ADM. In contrast to rat PMCs, RBL-2H3 cells were shown to also express RDC-1 to which CGRP can bind more firmly than ADM, although mRNA the other CGRP receptor, CRLR/RAMP1, was not detected.

These observations in the current study that RBL-2H3 cells are positive for mRNA of not only ADM but also CGRP receptors are of interest given the roles of these peptides in physiological and pathophysiological conditions in which mast cells, especially MMC, are involved. ADM is secreted from vascular endothelial cells and smooth muscle cells, ${ }^{4,5)}$ and has a role in potent vasodilation. ${ }^{1,3)}$ CGRP is localized in and released from sensory nerves as a neurotransmitter when these nerves are activated as in asthma and allergy. ${ }^{2,16)}$ On the other hand, MMC is distributed in the mucosa of the gastrointestinal tract and in the lamina propria of the respiratory tract, ${ }^{12)}$ where ADM and CGRP are richly present in the microvasculature and sensory nerve systems. Therefore, it is possible that the interaction of mast cells with these peptides plays a role as a component of innate immunity in the host defense against bacterial and parasitical infection as well as having a role in allergic diseases.

\section{REFERENCES}

1) Wimalawansa S. J., Crit. Rev. Neurobiol., 11, 167-239 (1997).

2) Springer J., Geppetti P., Fischer A., Groneberg D. A., Pulm. Pharm. Ther., 16, 121-130 (2003).

3) Kitamura K., Kangawa K., Kawamoto M., Ichiki Y., Nakamura S., Matsuo H., Eto T., Biochem. Biophys. Res. Commun., 192, 553-560
(1993).

4) Kubo A., Minamino N., Isumi Y., Kangawa K., Dohi K., Matsuo H., FEBS Lett., 426, 233-237 (1998).

5) Kubo A., Minamino N., Isumi Y., Katafuchi T., Kangawa K., Dohi K., Matsuo H., J. Biol. Chem., 273, 16730-16738 (1998).

6) Kapas S., Clark A. J., Biochem. Biophys. Res. Commun., 217, 832838 (1995).

7) Juaneda C., Dumont Y., Quirion R., Trends Pharmacol. Sci., 21, 432 438 (2000).

8) Libert F., Parmentier M., Lefort A., Dinsart C., Van. Sande J., Maenhaut C., Simons M. J., Dumont J. E., Vassart G., Science, 244, 569572 (1989).

9) Collum R. G., Fisher P. E., Datta M., Mellis S., Thiele C., Huebner K., Croce C. M., Israel M. A., Nucleic Acids Res., 20, 4919-4925 (1992).

10) McLatchie L. M., Fraser N. J., Main M. J., Wise A., Brown J., Thompson N., Solari R., Lee M. G., Foord S. M., Nature (London), 393, 333 -339 (1998).

11) Boyce J. A., J. Allergy Clin. Immunol., 111, 24-32 (2003).

12) Welle M., J. Leukoc. Biol., 61, 233-245 (1997).

13) Seldin D. C., Adelman S., Austen K. F., Stevens R. L., Hein A., Caulfield J. P., Woodbury R. G., Proc. Natl. Acad. Sci. U.S.A., 82, 3871-3875 (1985).

14) Penner R., Matthews G., Neher E., Nature (London), 334, 499—504 (1988).

15) Lowman M. A., Benyon R. C., Church M. K., Br. J. Pharmacol., 98, $121-130$ (1988).

16) Forsythe P., McGarvey L. P., Heaney L. G., MacMahon J., Ennis M., Clin. Exp. Allergy, 30, 225-232 (2000).

17) Ottosson A., Edvinsson L., Cephalalgia, 17, 166-174 (1997).

18) Yoshida M., Yoshida H., Kitaichi K., Hiramatsu K., Kimura T., Ito Y., Kume H., Yamaki K., Suzuki R., Shibata E., Hasegawa T., Takagi K., Regul. Pept., 101, 163-168 (2001).

19) Matsushima M., Kitaichi K., Tatsumi Y., Hasegawa T., Takagi K., Eur. J. Pharmacol., 464, 111-114 (2003).

20) Yamashita M., Kushihara M., Hirasawa N., Takasaki W., Takahagi H., Takayanagi M., Ohuchi K., Br. J. Pharmacol., 129, 367-373 (2000).

21) Qing X., Svaren J., Keith I. M., Am. J. Physiol. Lung. Cell Mol. Physiol., 280, L547-L554 (2001).

22) Robbins M., McKinney M., Brain Res. Mol. Brain Res., 13, 83-92 (1992). 